\title{
Erratum to: Gravitational waves from colliding vacuum bubbles in gauge theories
}

\author{
Marek Lewicki $^{1, \mathrm{a}}$, Ville Vaskonen ${ }^{2, \mathrm{~b}}{ }_{\mathbb{C}}$ \\ ${ }^{1}$ Faculty of Physics, University of Warsaw, ul. Pasteura 5, 02-093 Warsaw, Poland \\ ${ }^{2}$ Institut de Fisica d'Altes Energies (IFAE), The Barcelona Institute of Science and Technology, Campus UAB, 08193 Bellaterra, Barcelona, Spain
}

Received: 17 November 2021 / Accepted: 28 November 2021 / Published online: 7 December 2021

(C) The Author(s) 2021

\section{Erratum to: Eur. Phys. J. C (2021) 81:437 https://doi.org/10.1140/epjc/s10052-021-09232-3}

In this Erratum we correct the numerical results of the thinwall simulations given in the article.

As shown in the article, the abundance of GWs produced in bubble collisions in a logarithmic frequency interval can be written as

$\Omega_{\mathrm{GW}}(\omega) \equiv \frac{1}{E_{\mathrm{tot}}} \frac{\mathrm{d} E}{\mathrm{~d} \ln \omega}=\left(\frac{H}{\beta}\right)^{2}\left(\frac{\alpha}{1+\alpha}\right)^{2} S(\omega)$,

We calculate $S$ by performing thin-wall simulations where we nucleate bubbles according to the rate $\Gamma \propto e^{\beta t}$ in a cubic box of size $(7 / \beta)^{3}$ with periodic boundary conditions. We perform the angular integrals over the bubble surfaces by discretising each of them with $10^{6}$ evenly distributed points. Our results are calculated from 60 simulations. In the same way as in the article, we parametrize the results as a broken power-law,

$S_{\mathrm{fit}}(\omega)=\frac{\bar{S}(a+b)^{c}}{\left[b\left(\frac{\omega}{\bar{\omega}}\right)^{-a / c}+a\left(\frac{\omega}{\bar{\omega}}\right)^{b / c}\right]^{c}}$,

where $\bar{S}$ and $\bar{\omega}$ are the peak amplitude and angular frequency of the spectrum, $a, b>0$ are the low- and high-frequency slopes of the spectrum respectively, and $c$ determines the width of the peak. We show the parameter values and their errors resulting from fits to our simulation results in Table 1 for $\xi=2,3,4$ and the envelope approximation and illustrate these fits in Fig. 1. In the article, the table and the corre-

The original article can be found online at https://doi.org/10.1140/ epjc/s10052-021-09232-3.

\footnotetext{
a e-mail: marek.lewicki@fuw.edu.pl

b e-mail: vvaskonen@ifae.es (corresponding author)
}

Table 1 Fitted values for the parametrization of the spectral shape (2)

\begin{tabular}{lccccc}
\hline & $100 \bar{S}$ & $\bar{\omega} / \beta$ & $a$ & $b$ & $c$ \\
\hline$\xi=2$ & $4.23 \pm 0.1$ & $0.68 \pm 0.01$ & $1.00 \pm 0.02$ & $2.17 \pm 0.05$ & $2.02 \pm 0.1$ \\
$\xi=3$ & $3.61 \pm 0.1$ & $0.82 \pm 0.01$ & $2.34 \pm 0.03$ & $2.41 \pm 0.02$ & $4.20 \pm 0.2$ \\
$\xi=4$ & $3.46 \pm 0.1$ & $0.93 \pm 0.01$ & $2.87 \pm 0.04$ & $2.42 \pm 0.02$ & $4.63 \pm 0.2$ \\
env. & $2.75 \pm 0.1$ & $1.72 \pm 0.04$ & $2.98 \pm 0.02$ & $1.01 \pm 0.02$ & $2.18 \pm 0.1$
\end{tabular}

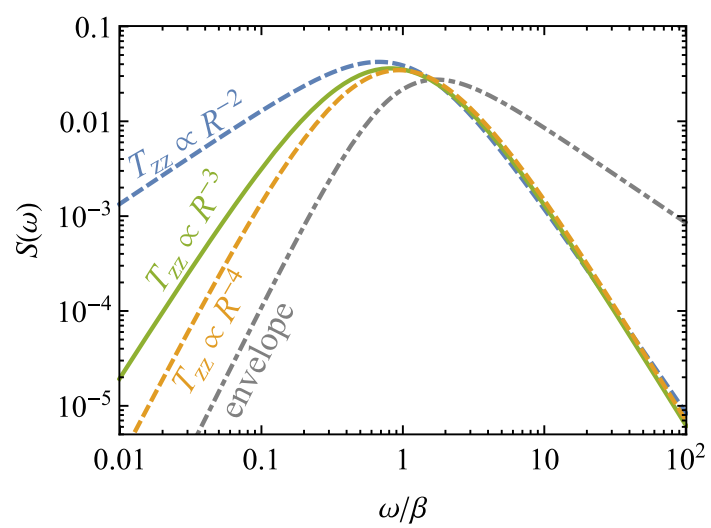

Fig. 1 The spectral shape of GWs (see Eq. (1)) from vacuum bubble collisions. The curves show broken power-law fits to the simulation results for different decay-laws of the GW source after collisions and in the envelope approximation. The solid curve is realised in the case of breaking of a gauge U(1) symmetry. The corresponding parameters of the fit, and their errors, are given in Table 1

sponding plot are wrong due to a small error in the numerical simulation.

Open Access This article is licensed under a Creative Commons Attribution 4.0 International License, which permits use, sharing, adaptation, distribution and reproduction in any medium or format, as long as you 
give appropriate credit to the original author(s) and the source, provide a link to the Creative Commons licence, and indicate if changes were made. The images or other third party material in this article are included in the article's Creative Commons licence, unless indicated otherwise in a credit line to the material. If material is not included in the article's Creative Commons licence and your intended use is not permitted by statutory regulation or exceeds the permitted use, you will need to obtain permission directly from the copyright holder. To view a copy of this licence, visit http://creativecomm ons.org/licenses/by/4.0/.

Funded by $\mathrm{SCOAP}^{3}$. 